\title{
Stability analysis of switching hyperbolic systems: the example of SMB chromatography
}

\author{
Georges Bastin, Jean-Michel Coron and Brigitte d'Andréa-Novel, \\ Paul Suvarov, Alain Vande Wouwer and Achim Kienle
}

\begin{abstract}
This paper addresses the issue of the exponential stability of hybrid hyperbolic systems with switching boundary conditions. Our contribution is to show, through the specific example of SMB chromatography, how exponential stability (in $L_{2}$-norm) can be established when the switching mechanism is precisely defined. The obtained stability conditions are direct generalizations of the corresponding results for the unswitched case.
\end{abstract}

\section{INTRODUCTION}

The exponential stability of systems of conservation laws over a bounded domain in one spatial dimension is a research topic which has given rise to a significant amount of activity in the recent years (see [3] [4], [5] [6] [7] [13] [17] among many other relevant references).

In certain practical applications, it is of interest to address situations where the system exhibits periodic time switching between various sets of boundary conditions. From the viewpoint of exponential stability analysis, a system of conservation laws with switching boundary conditions can be viewed as a hybrid system on an infinite dimensional state space. While hybrid systems based on ordinary differential equations are extensively considered in the literature (e.g. [8], [14], [15]), hybrid systems based on partial differential equations are relatively unexplored.

As emphasized in [9], two fundamental stability issues are of interest for switching systems:

- Issue A: Find conditions for which the switched system is asymptotically stable for any switching signal.

GB and JMC are partially supported by the ERC advanced grant 266907 (CPDENL) of the 7th Research Framework Programme (FP7). GB, PS and AVW are partially supported by the Belgian Programme on Interuniversity Attraction Poles (IAP VII/19).

G. Bastin is with the Department of Mathematical Engineering, ICTEAM, Université catholique de Louvain, 1348 Louvain-la-Neuve, Belgium. georges.bastineuclouvain.be

J-M. Coron is with the Laboratoire Jacques-Louis Lions, Institut Universitaire de France, Université Pierre et Marie Curie, 75252 Paris, France. coroneann. jussieu.fr

B. d'Andréa-Novel is with the Centre de Robotique (CAOR), Ecole Nationale Supérieure des Mines de Paris, 75272 Paris, France brigitte.dandrea-novel@mines-paristech.fr

P. Suvarov is with the Service dAutomatique, Université de Mons, 7000 Mons, Belgium and the Max-Planck-Institut fur Dynamik Komplexer Technisher Systeme, 39106 Magdeburg, Germanypaul . suvarov@umons.ac.be

A. Vande Wouwer are with Service dAutomatique, Université de Mons, 7000 Mons, Belgium alain.vandewouwer@umons.ac.be

A. Kienle is with the Institut fur Automatisierungstechnik, Ottovon-Guericke-Universitt and the Max-Planck-Institut fur Dynamik Komplexer Technisher Systeme, 39106 Magdeburg, Germany kienle@mpi-magdeburg.mpg.de
- Issue B: Show that the switched system is asymptotically stable for a given switching strategy or a class of switching strategies.

Using the method of characteristics, a recent paper by Amin et al. [1] deals with Issue A for switched hyperbolic systems and gives sufficient boundary conditions for the exponential stability (in $H_{\infty}$-norm) under arbitrary switching signals. As said by the authors of that paper, this kind of analysis is "relevant when the switching mechanism is either unknown or too complicated for a more careful stability analysis". In this paper our purpose is to address the Issue B through the specific example of SMB chromatography [16] and to show how exponential stability (in $L_{2}$-norm) can be established when the switching mechanism is precisely defined. The obtained stability conditions are direct generalizations of the corresponding results for the unswitched case.

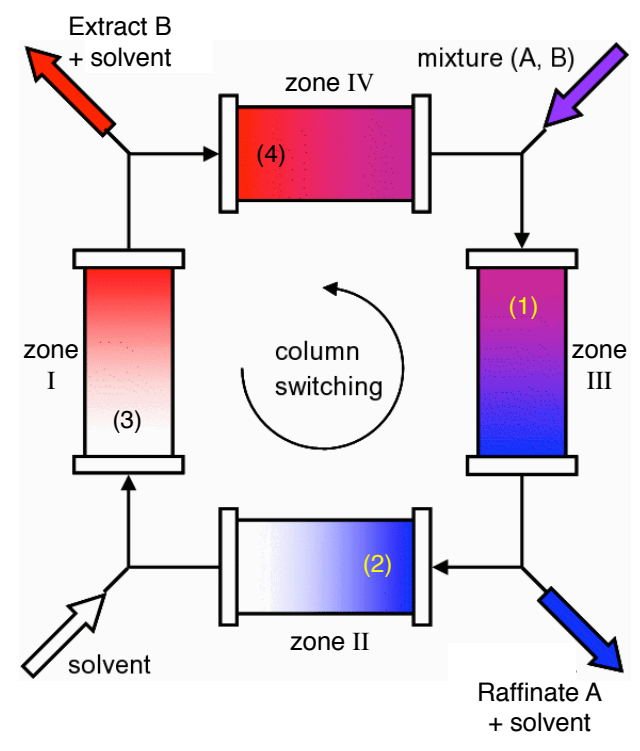

Fig. 1. SMB chromatography.

\section{MODELLING}

Simulated moving bed (SMB) chromatography is a technology where several interconnected chromatographic columns are switched periodically against the fluid flow. This allows for a continuous separation with a better performance than the discontinuous single-column chromatography. A standard SMB chromatography process is represented in Fig.1. The input flows (feed mixture and solvent) and output flows (extract and raffinate) divide the system in four zones 
each containing one chromatographic column. The four (mobile) columns are labeled 1,2,3,4. The four operating zones are labeled I, II, III, IV. Pumps connected at each port determine the liquid phase flow rates in all the zones. The feed mixture composed of species A and B is injected between zone II and zone III. The adsorbent is chosen in such a way that the two components are adsorbed at different rates, allowing them to travel with different velocities. The less adsorbed component (A) is collected at the raffinate port and the more adsorbed one (B) at the extract port. The separation of the two components is performed in zones II and III, whereas zones I and IV are dedicated to adsorbent regeneration and solvent recycling. A liquid-solid counter-current movement is obtained by a periodic circular switching of the columns in the opposite direction to the liquid phase flow as shown in Fig.1.

We introduce the following notations :

- The switching time period is $T$ and the column length is $L$.

- $C_{i}^{\ell}(t, x) \geqslant 0,0 \leqslant x \leqslant L, t \geqslant 0$, is the concentration of species $\ell \in\{A, B\}$ in the column $i \in\{1,2,3,4\}$,

- $V_{\mathrm{I}}$ is the fluid velocity in the columns located in zones I and III, $V_{\text {II }}$ is the fluid velocity in the columns located in zones II and IV with $V_{\mathrm{I}}>V_{\text {II }}>0$,

- $h_{A}>0$ and $h_{B}>0$ are the Henry coefficients,

- $F$ is the phase ratio, i.e. $F=(1-\epsilon) / \epsilon$ where $\epsilon \in(0,1)$ is the bed porosity.

In this paper, we limit ourselves to the case of linear adsorption isotherms which is, for instance, a plausible model for the separation of Fructo-OligoSaccharides [12]. We first state the dynamical model during the first time period, assuming that column 1 is in zone III, column 2 in zone IV, column 3 in zone I and column 4 in zone II, as shown in Fig.1. The process dynamics are represented by the following set of conservation laws:

For $0 \leqslant t<T$,

$$
\begin{aligned}
& \left(1+F h_{A}\right) \partial_{t} C_{1}^{A}+V_{\mathbf{I}} \partial_{x} C_{1}^{A}=0, \\
& \left(1+F h_{A}\right) \partial_{t} C_{2}^{A}+V_{\mathbf{I I}} \partial_{x} C_{2}^{A}=0, \\
& \left(1+F h_{A}\right) \partial_{t} C_{3}^{A}+V_{\mathbf{I}} \partial_{x} C_{3}^{A}=0, \\
& \left(1+F h_{A}\right) \partial_{t} C_{4}^{A}+V_{\mathbf{I I}} \partial_{x} C_{4}^{A}=0, \\
& \left(1+F h_{B}\right) \partial_{t} C_{1}^{B}+V_{\mathbf{I}} \partial_{x} C_{1}^{B}=0, \\
& \left(1+F h_{B}\right) \partial_{t} C_{2}^{B}+V_{\mathbf{I I}} \partial_{x} C_{2}^{B}=0 \\
& \left(1+F h_{B}\right) \partial_{t} C_{3}^{B}+V_{\mathbf{I}} \partial_{x} C_{3}^{B}=0, \\
& \left(1+F h_{B}\right) \partial_{t} C_{4}^{B}+V_{\mathbf{I I}} \partial_{x} C_{4}^{B}=0 .
\end{aligned}
$$

The boundary conditionsare the expression of the conservation of flows the equality of concentrations at the junctions of the columns:

$$
\begin{aligned}
V_{\mathrm{I}} C_{1}^{A}(t, 0) & =V_{\mathrm{II}} C_{4}^{A}(t, L)+V_{F} C_{F}^{A}, \\
C_{2}^{A}(t, 0) & =C_{1}^{A}(t, L), \\
V_{\mathrm{I}} C_{3}^{A}(t, 0) & =V_{\mathrm{II}} C_{2}^{A}(t, L), \\
C_{4}^{A}(t, 0) & =C_{3}^{A}(t, L) . \\
V_{\mathrm{I}} C_{1}^{B}(t, 0) & =V_{\mathrm{II}} C_{4}^{B}(t, L)+V_{F} C_{F}^{B}, \\
C_{2}^{B}(t, 0) & =C_{1}^{B}(t, L), \\
V_{\mathrm{I}} C_{3}^{B}(t, 0) & =V_{\mathrm{II}} C_{2}^{B}(t, L), \\
C_{4}^{B}(t, 0) & =C_{3}^{B}(t, L) .
\end{aligned}
$$

In these equations, $V_{F}>0$ is the constant fluid velocity while $C_{F}^{A}>0, C_{F}^{B}>0$ are the constant species concentrations in the input flow.

We introduce the following vector and matrix notations:

$$
\begin{gathered}
\mathbf{C}^{\ell}(t, x) \triangleq\left(C_{1}^{\ell}(t, x), C_{2}^{\ell}(t, x), C_{3}^{\ell}(t, x), C_{4}^{\ell}(t, x)\right)^{T}, \\
U^{\ell} \triangleq\left(\left(V_{F} / V_{I}\right) C_{F}^{\ell}, 0,0,0\right)^{T}, \quad \ell \in\{A, B\}, \\
\mathbf{C}(t, x)=\left(\begin{array}{l}
\mathbf{C}^{A}(t, x) \\
\mathbf{C}^{B}(t, x)
\end{array}\right),
\end{gathered}
$$$$
\Upsilon \triangleq \operatorname{diag}\left\{V_{\mathrm{I}}, V_{\mathrm{II}}, V_{\mathrm{I}}, V_{\mathrm{II}}\right\} \text {, }
$$

$$
\mathbf{K} \triangleq\left(\begin{array}{cccc}
0 & 0 & 0 & V_{\text {II }} / V_{\mathbf{I}} \\
1 & 0 & 0 & 0 \\
0 & V_{\text {II }} / V_{\mathrm{I}} & 0 & 0 \\
0 & 0 & 1 & 0
\end{array}\right) .
$$

With these notations, the model equations are written in compact form as follows:

$$
\begin{gathered}
0 \leqslant t<T, \quad \ell \in\{A, B\}, \\
\left(1+F h_{\ell}\right) \partial_{t} \mathbf{C}^{\ell}+\Upsilon \partial_{x} \mathbf{C}^{\ell}=0, \\
\mathbf{C}^{\ell}(t, 0)=\mathbf{K} \mathbf{C}^{\ell}(t, L)+U^{\ell} .
\end{gathered}
$$

We now consider the second time period when the columns have been shifted by one position such that column 1 is now located in zone II, column 2 in zone III, etc ... To take the shifting process into account in a systematic way, we introduce the following permutation matrix:

$$
P=\left(\begin{array}{llll}
0 & 0 & 0 & 1 \\
1 & 0 & 0 & 0 \\
0 & 1 & 0 & 0 \\
0 & 0 & 1 & 0
\end{array}\right) .
$$

Then, it can be checked that the model equations during the second period become

$$
\begin{gathered}
T \leqslant t<2 T, \quad \ell \in\{A, B\} \\
\left(1+F h_{\ell}\right) \partial_{t} \mathbf{C}^{\ell}+P \Upsilon P^{T} \partial_{x} \mathbf{C}^{\ell}=0 \\
\mathbf{C}^{\ell}(t, 0)=P \mathbf{K} P^{T} \mathbf{C}^{\ell}(t, L)+P U^{\ell} .
\end{gathered}
$$


It is then clear that, by iteration, we have the following general form for the hyperbolic system of conservation laws describing the periodic SMB chromatography process:

$$
\begin{gathered}
m T \leqslant t<(m+1) T, \quad m=0,1,2,3,4,5, \ldots, \quad \ell \in\{A, B\}, \\
\left(1+F h_{\ell}\right) \partial_{t} \mathbf{C}^{\ell}+\left(P^{m}\right) \Upsilon\left(P^{m}\right)^{T} \partial_{x} \mathbf{C}^{\ell}=0, \\
\mathbf{C}^{\ell}(t, 0)=\left(P^{m}\right) \mathbf{K}\left(P^{m}\right)^{T} \mathbf{C}^{\ell}(t, L)+\left(P^{m}\right) U^{\ell} .
\end{gathered}
$$

In this paper, we consider the case where, under the periodic operation described above, the system (1) has a single steady-state periodic solution $\mathbf{C}^{*} \triangleq\left(\mathbf{C}^{* A}, \mathbf{C}^{* B}\right)$ such that $\mathbf{C}^{*}(t, x)=\mathbf{C}^{*}(t+4 T, x), x \in[0, L], t \geqslant 0$. Our concern is to analyze the exponential stability of this steady-state.

\section{EXPONENTIAL STABILITY}

In order to put the system in characteristic form, we define the Riemann coordinates:

$$
\begin{aligned}
& R_{i}^{A}=\left(1+F h_{A}\right)\left(C_{i}^{A}-C_{i}^{* A}\right), \\
& R_{i}^{B}=\left(1+F h_{B}\right)\left(C_{i}^{B}-C_{i}^{* B}\right), \quad i=1,2,3,4 .
\end{aligned}
$$

In these Riemann coordinates, the periodic linear system is written

$$
\begin{gathered}
m T \leqslant t<(m+1) T, \quad m=0,1,2,3,4,5, \ldots, \quad \ell \in\{A, B\} \\
\partial_{t} \mathbf{R}^{\ell}+\Lambda_{m}^{\ell} \partial_{x} \mathbf{R}^{\ell}=0, \\
\mathbf{R}^{\ell}(t, 0)=\mathbf{K}_{m} \mathbf{R}^{\ell}(t, L) .
\end{gathered}
$$

with the following notations:

$$
\begin{gathered}
\Lambda^{\ell} \triangleq \operatorname{diag}\left\{\lambda_{1}^{\ell}, \lambda_{2}^{\ell}, \lambda_{1}^{\ell}, \lambda_{2}^{\ell}\right\} \\
\text { with } \quad \lambda_{1}^{\ell} \triangleq \frac{V_{\mathbf{I}}}{1+F h_{\ell}}, \quad \lambda_{2}^{\ell} \triangleq \frac{V_{\text {II }}}{1+F h_{\ell}}, \\
\Lambda_{m}^{\ell} \triangleq\left(P^{m}\right) \Lambda^{\ell}\left(P^{m}\right)^{T}, \quad \mathbf{K}_{m}=\left(P^{m}\right) \mathbf{K}\left(P^{m}\right)^{T} .
\end{gathered}
$$

We have the following stability property.

Theorem 1. The periodic solution $\mathbf{C}^{*}(t, x)$ of the system (1) is exponentially stable if

$$
T>\frac{L}{\lambda_{2}^{\ell}}-\frac{L}{\lambda_{1}^{\ell}}, \quad \ell \in\{A, B\} .
$$

Proof: As advocated in [2] for the analysis of hybrid systems, we follow a so-called "multiple Lyapunov function" approach with the two following candidate quadratic Lyapunov functions:

$\mathbf{V}_{1} \triangleq$

$$
\begin{array}{r}
\sum_{\ell \in\{A, B\}} \int_{0}^{L}\left\{\frac{p_{1}}{\lambda_{1}^{\ell}}\left(\left[R_{1}^{\ell}(t, x)\right]^{2}+\left[R_{3}^{\ell}(t, x)\right]^{2}\right) \exp \left(-\frac{\mu x}{\lambda_{1}^{\ell}}\right)\right. \\
\left.+\frac{p_{2}}{\lambda_{2}^{\ell}}\left(\left[R_{2}^{\ell}(t, x)\right]^{2}+\left[R_{4}^{\ell}(t, x)\right]^{2}\right) \exp \left(-\frac{\mu x}{\lambda_{2}^{\ell}}\right)\right\} d x
\end{array}
$$

$\mathbf{V}_{2} \triangleq$

$$
\begin{array}{r}
\sum_{\ell \in\{A, B\}} \int_{0}^{L}\left\{\frac{p_{2}}{\lambda_{2}^{\ell}}\left(\left[R_{1}^{\ell}(t, x)\right]^{2}+\left[R_{3}^{\ell}(t, x)\right]^{2}\right) \exp \left(-\frac{\mu x}{\lambda_{2}^{\ell}}\right)\right. \\
\left.+\frac{p_{1}}{\lambda_{1}^{\ell}}\left(\left[R_{2}^{\ell}(t, x)\right]^{2}+\left[R_{4}^{\ell}(t, x)\right]^{2}\right) \exp \left(-\frac{\mu x}{\lambda_{1}^{\ell}}\right)\right\} d x
\end{array}
$$

with positive constant coefficients $p_{1}, p_{2}$ and $\mu$. The time derivatives of $\mathbf{V}_{1}$ and $\mathbf{V}_{2}$ along the trajectories of the system (2) are

$$
m=0,2,4, \ldots
$$

For $m T \leqslant t<(m+1) T$,

$$
\begin{aligned}
& \frac{d \mathbf{V}_{1}}{d t}=-\mu \mathbf{V}_{1} \\
&-\sum_{\ell \in\{A, B\}}\left\{\left(p_{1} \exp \left(-\frac{\mu L}{\lambda_{1}^{\ell}}\right)-p_{2}\right)\right. \\
&\left.\left(\left[R_{1}^{\ell}(t, L)\right]^{2}+\left[R_{3}^{\ell}(t, L)\right]^{2}\right)\right\} \\
&-\sum_{\ell \in\{A, B\}}\left\{\left(p_{2} \exp \left(-\frac{\mu L}{\lambda_{2}^{\ell}}\right)-\left(\frac{\lambda_{2}^{\ell}}{\lambda_{1}^{\ell}}\right)^{2} p_{1}\right)\right. \\
&\left.\left(\left[R_{2}^{\ell}(t, L)\right]^{2}+\left[R_{4}^{\ell}(t, L)\right]^{2}\right)\right\}
\end{aligned}
$$

For $(m+1) T \leqslant t<(m+2) T$,

$$
\begin{aligned}
& \frac{d \mathbf{V}_{2}}{d t}=-\mu \mathbf{V}_{2} \\
&-\sum_{\ell \in\{A, B\}}\left\{\left(p_{1} \exp \left(-\frac{\mu L}{\lambda_{1}^{\ell}}\right)-p_{2}\right)\right. \\
&\left.\left(\left[R_{2}^{\ell}(t, L)\right]^{2}+\left[R_{4}^{\ell}(t, L)\right]^{2}\right)\right\} \\
&-\sum_{\ell \in\{A, B\}}\left\{\left(p_{2} \exp \left(-\frac{\mu L}{\lambda_{2}^{\ell}}\right)-\left(\frac{\lambda_{2}^{\ell}}{\lambda_{1}^{\ell}}\right)^{2} p_{1}\right)\right. \\
&\left.\left(\left[R_{1}^{\ell}(t, L)\right]^{2}+\left[R_{3}^{\ell}(t, L)\right]^{2}\right)\right\} .
\end{aligned}
$$

If the parameters $p_{1}$ and $p_{2}$ are selected such that

$$
1<\frac{p_{1}}{p_{2}}<\left(\frac{\lambda_{1}^{\ell}}{\lambda_{2}^{\ell}}\right)^{2}=\left(\frac{V_{\mathrm{I}}}{V_{\mathrm{II}}}\right)^{2}
$$

then $\mu>0$ can be selected such that

$$
\frac{p_{1}}{p_{2}} \exp \left(-\frac{\mu L}{\lambda_{1}^{\ell}}\right)>1, \quad \frac{p_{2}}{p_{1}}\left(\frac{\lambda_{1}^{\ell}}{\lambda_{2}^{\ell}}\right)^{2} \exp \left(-\frac{\mu L}{\lambda_{2}^{\ell}}\right)>1,
$$


which imply

$$
\begin{aligned}
& m T \leqslant t<(m+1) T, \\
& \frac{d \mathbf{V}_{1}}{d t} \leqslant-\mu \mathbf{V}_{1} \Rightarrow \mathbf{V}_{1}((m+1) T) \leqslant \mathbf{V}_{1}(m T) e^{-\mu T}, \\
& (m+1) T \leqslant t<(m+2) T, \\
& \frac{d \mathbf{V}_{2}}{d t} \leqslant-\mu \mathbf{V}_{2} \Rightarrow \mathbf{V}_{2}((m+2) T) \leqslant \mathbf{V}_{2}((m+1) T) e^{-\mu T} .
\end{aligned}
$$

Let us select $p_{1} / p_{2}$ as follows:

$$
\frac{p_{1}}{p_{2}}=\frac{V_{\mathrm{I}}}{V_{\mathrm{II}}}>1 .
$$

Let us define a parameter $\alpha>0$ selected such that

$$
\frac{1}{T}\left(\frac{L}{\lambda_{2}^{\ell}}-\frac{L}{\lambda_{1}^{\ell}}\right)<\frac{\ln \alpha}{\mu T}<1 .
$$

Using inequalities (6) and (7), we then have

$$
\alpha \geqslant \frac{\exp \left(-\frac{\mu L}{\lambda_{1}^{\ell}}\right)}{\exp \left(-\frac{\mu L}{\lambda_{2}^{\ell}}\right)} \geqslant \frac{\frac{p_{1}}{\lambda_{1}^{\ell}} \exp \left(-\frac{\mu x}{\lambda_{1}^{\ell}}\right)}{\frac{p_{2}}{\lambda_{2}^{\ell}} \exp \left(-\frac{\mu x}{\lambda_{2}^{\ell}}\right)}>1 .
$$

By combining this inequality with the definitions of $\mathbf{V}_{1}$ and $\mathbf{V}_{2}$, it can be checked that

$$
\frac{1}{\alpha} \mathbf{V}_{2} \leqslant \mathbf{V}_{1} \leqslant \alpha \mathbf{V}_{2}, \quad \forall t, \forall x
$$

From (4), (5) and (8), we then have:

$$
\begin{gathered}
\mathbf{V}_{1}((m+2) T) \leqslant \alpha \mathbf{V}_{2}((m+2) T) \leqslant \alpha e^{-\mu T} \mathbf{V}_{2}((m+1) T) \\
\leqslant \alpha^{2} e^{-\mu T} \mathbf{V}_{1}((m+1) T) \leqslant\left(\alpha e^{-\mu T}\right)^{2} \mathbf{V}_{1}(m T)
\end{gathered}
$$

Mutatis mutandis, obviously we also have

$$
\mathbf{V}_{2}((m+3) T) \leqslant\left(\alpha e^{-\mu T}\right)^{2} \mathbf{V}_{2}((m+1) T)
$$

Now, from (7) we have:

$$
\alpha e^{-\mu T}<1
$$

Therefore, $\mathbf{V}_{1}(t)$ and $\mathbf{V}_{2}(t)$ exponentially converge to zero and the periodic time solution $\mathbf{C}^{*}$ is exponentially stable.

\section{SIMULATION EXPERIMENT}

In this section we present a simulation experiment of a SMB process implemented under the operating conditions reported in [11] for the separation of fructo-oligosaccharides.

The parameter values are:

$$
\begin{aligned}
& L=0.248 \mathrm{~m}, \quad T=114.95 \mathrm{~s}, \\
& V_{\mathrm{I}}=0.036 \mathrm{~m} / \mathrm{s}, \quad V_{\mathrm{II}}=0.022 \mathrm{~m} / \mathrm{s}, \\
& h_{A}=0.3954, \quad h_{B}=0.0251, \\
& C_{F}^{A}=64 \mathrm{mg} / \mathrm{ml}, \quad C_{F}^{B}=85 \mathrm{mg} / \mathrm{ml}, \\
& \epsilon=0.378 \quad F=\frac{1-\epsilon}{\epsilon}=1.6427 .
\end{aligned}
$$

From these values, we verify that the stability condition of Theorem 1 is satisfied since

$$
\begin{aligned}
& T>\frac{L}{\lambda_{2}^{A}}-\frac{L}{\lambda_{1}^{A}} \approx 67 \mathrm{~s}, \\
& T>\frac{L}{\lambda_{2}^{B}}-\frac{L}{\lambda_{1}^{B}} \approx 42 \mathrm{~s} .
\end{aligned}
$$

We simulate the start-up of the process from zero initial conditions (i.e. zero initial concentrations in the columns). The simulation results are shown in Fig.2 and Fig.3. We see in Fig. 2 that the steady-state periodic regime is reached within about 10 column shifts (i.e. 2.5 rounds).

It may also be observed in Fig.3 that the separation between species A and B is effective but not perfect. This is an inherent limitation of SMB processes implemented with four columns as considered in this paper for simplicity. In order to reach a total purity of the separation, industrial SMB processes are generally implemented with eight (e.g. [16]) or even twelve columns (e.g. [10]).

\section{CONCLUSiOnS}

In this communication, we have been concerned with the exponential stability of hybrid hyperbolic systems with switching boundary conditions. Our contribution has been to show how the exponential stability (in $L_{2}$-norm) can be established in the special case of SMB chromatography. The approach which has been followed is a direct generalisation of the method for the unswitched case. It is based on the fact that the two underlying subsystems are exponentially stable with different quadratic Lyapunov functions. The convergence of the hybrid system is then established by switching between these two Lyapunov functions in synchronization with the process physical switching.

\section{REFERENCES}

[1] S. Amin, F. Hante, and A. Bayen. Exponential stability of switched linear hyperbolic initial-boundary value problems. IEEE Transactions on Automatic Control, 56(2):291-301, February 2012.

[2] M.-S. Branicky. Multiple Lyapunov theory and other analysis tools for switched and hybrid systems. IEEE Transactions on Automatic Control, 43(4):475-482, 1998.

[3] J-M. Coron, G. Bastin, and B. d'Andréa-Novel. Dissipative boundary conditions for one dimensional nonlinear hyperbolic systems. SIAM Journal of Control and Optimization, 47(3):1460-1498, 2008.

[4] A. Diagne, G. Bastin, and J-M. Coron. Lyapunov exponential stability of linear hyperbolic systems of balance laws. Automatica, 48:109-114, 2012.

[5] M. Dick, M. Gugat, and G. Leugering. Classical solutions and feedback stabilisation for the gas flow in a sequence of pipes. Networks and Heterogeneous Media, 5(4):691-709, 2010.

[6] G. Leugering and J-P.G. Schmidt. On the modelling and stabilisation of flows in networks of open canals. SIAM Journal of Control and Optimization, 41(1):164-180, 2002.

[7] T-T. Li. Global Classical Solutions for Quasi-Linear Hyperbolic Systems. Research in Applied Mathematics. Masson and Wiley, 1994.

[8] D. Liberzon. Switching in Systems and Control. Systems and Control: Foundations and Applications. Birkhauser, 2003.

[9] D. Liberzon and A.S. Morse. Basic problems in stability and design of switched systems. IEEE Control Systems Magazine, pages 59-70, 1999. 


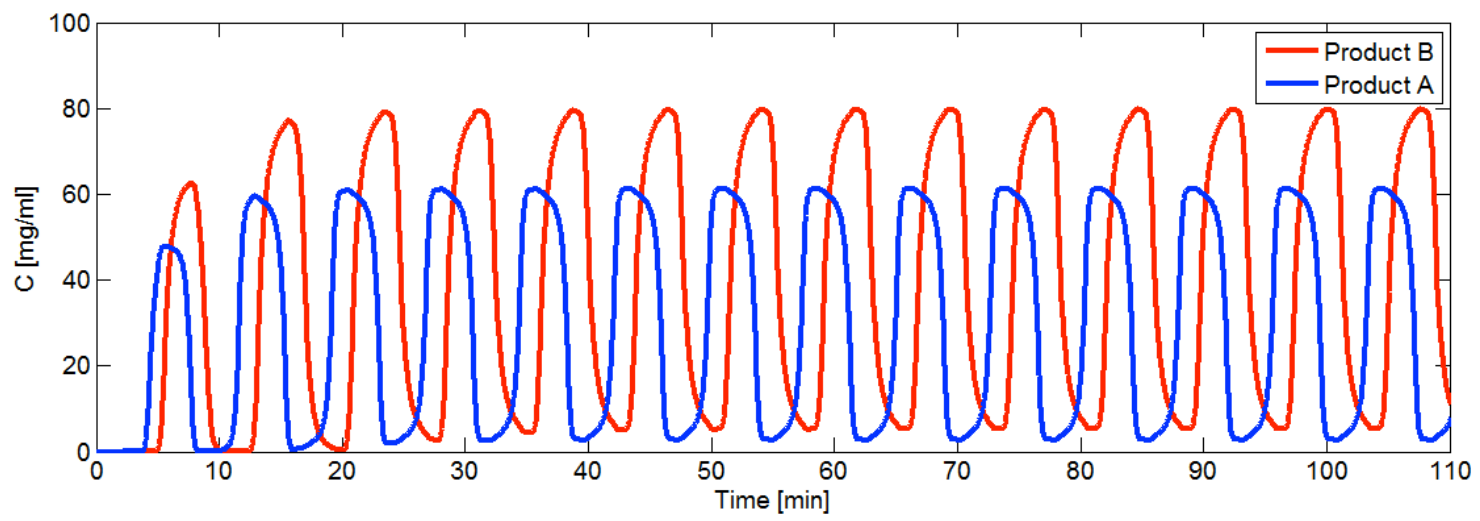

Fig. 2. Time evolution of the concentrations inside column 1 : exponential convergence towards the periodic regime.
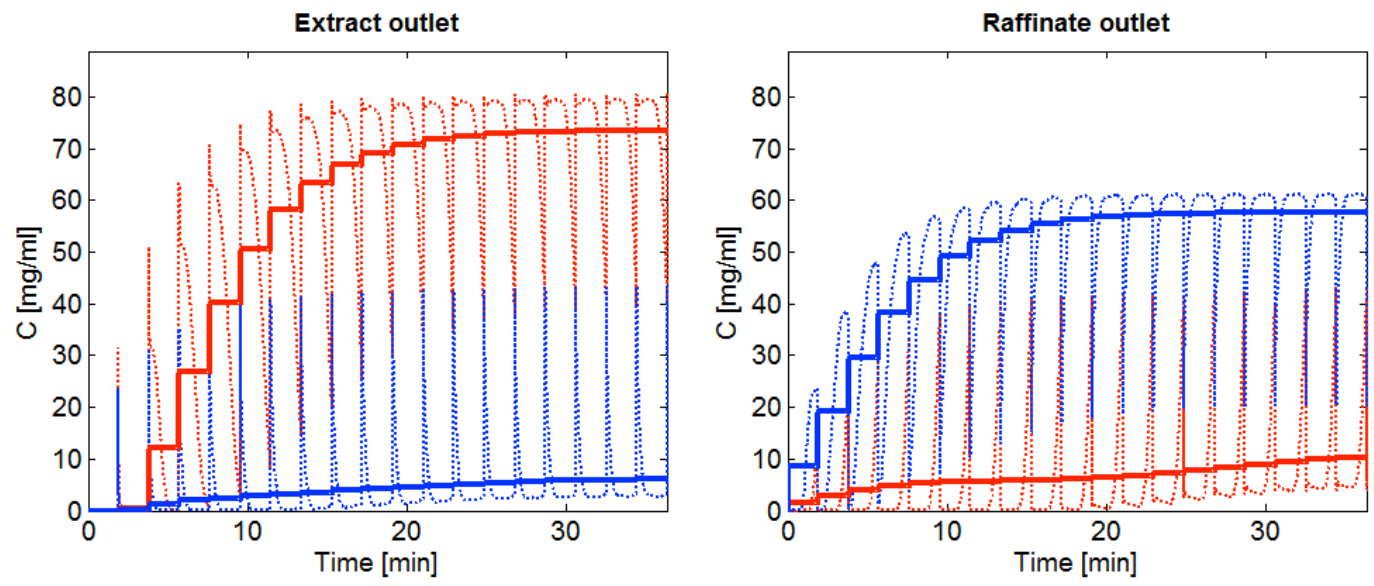

Fig. 3. Time evolution of the outlet concentrations (dotted line $=$ actual concentration, solid line $=$ average concentration).

[10] H. Lorenz, P. Sheehan, and A. Seidel-Morgenstern. Coupling of simulated moving bed chromatography and fractional crystallisation for efficient enantioseparation. Journal of Chromatography, 908:201214, 2001.

[11] C. Nobre, J.A. Teixeira, L. Rodrigues, A. Severino, C. Retamal, G. de Weireld, and A. Vande Wouwer. Operating conditions of a simulated moving bed chromatography unit for the purification of fructo-oligosaccharides. Technical report, University of Minho University of Mons, 2013.

[12] C. Nobre, J.A. Teixeira, and L.R. Rodrigues. Fructo-oligosaccharides purification from a fermentative broth using an activated charcoal column. New Biotechnology, 29(3):395-401, 2012.

[13] C. Prieur. Control of systems of conservation laws with boundary errors. Networks and Heterogeneous Media, 4(2):393-407, 2009.

[14] B. De Schutter and W. Heemels. Modeling and Control of Hybrid Systems. Delft Technical University, 2011.

[15] R. Shorten, F. Wirth, O. Mason, K. Wulff, and C. King. Stability criteria for switched and hybrid systems. SIAM Review, 49(4):545592, 2007.

[16] P. Suvarov, A. Vande Wouwer, and A. Kienle. A simple robust control for simulated moving bed chromatographic separation. In Proceedings IFAC Symposium on Advanced Control of Chemical processes, pages 137-142, Singapore, July, 10-13 2012.

[17] C-Z. Xu and G. Sallet. Exponential stability and transfer functions of processes governed by symmetric hyperbolic systems. ESAIM Control Optimisation and Calculus of Variations, 7:421-442, 2002. 